\title{
Hiperhomocisteinemia na insuficiência renal crônica
}

\author{
Hyperhomocysteinemia in chronic renal failure
}

\section{RE S U M O}

Fabiana Baggio NERBASS

Sérgio Antonio DRAIBE²

Lilian CUPPARI ${ }^{1,2}$

A homocisteína é um aminoácido sulfurado proveniente do metabolismo da metionina, cujo acúmulo anormal no plasma é um fator de risco para doenças vasculares, tanto na população em geral como nos pacientes com insuficiência renal crônica. Nestes, a prevalência de indivíduos com hiperhomocisteinemia é bastante elevada, mesmo na fase não dialítica da doença, em que a função renal está diminuída, mas ainda não é necessário tratamento dialítico. O principal fator que parece estar implicado na elevação dos níveis de homocisteína nestes pacientes com insuficiência renal crônica é a perda da massa renal, já que esta exerce uma importante função no metabolismo desse aminoácido. O tratamento da hiperhomocisteinemia na população em geral consiste na suplementação com as vitaminas envolvidas no seu metabolismo (folato, $B_{6}$ e $B_{12}$ ). Porém, em pacientes com insuficiência renal crônica, este tratamento não é completamente eficaz, pois apesar de promover a redução dos níveis de homocisteína, não alcança a normalização dos mesmos na maioria dos pacientes. Este estudo compreende uma revisão da etiologia da hiperhomocisteinemia na insuficiência renal crônica, sua relação com as doenças vasculares, seus principais determinantes e as formas de tratamento.

Termos de indexação: homocisteína, hiperhomocisteinemia, insuficiência renal crônica.

\section{A B S T R A C T}

Homocysteine is a sulfur-containing amino acid derived from the metabolism of methionine, whose abnormal accumulation in plasma is a risk factor for vascular disease in the general population and in patients with chronic renal disease. In these patients, the prevalence of individuals with hyperhomocysteinemia is very high, even in the pre-dialysis stage of the disease. The main factor that seems to be implicated on the elevation of homocysteine levels in this population is the renal mass loss, considering that the kidney has an important role in the metabolism of such amino acid. The treatment of hyperhomocysteinemia consists on supplementation of the vitamins that are involved in the homocysteine metabolism (folate, $B_{6}$ and $B_{12}$ ).

\footnotetext{
1 Pós-graduação em Nutrição, Universidade Federal de São Paulo, Escola Paulista de Medicina. São Paulo, SP, Brasil.

2 Disciplina de Nefrologia, Universidade Federal de São Paulo, Escola Paulista de Medicina. Rua Pedro de Toledo, 282, Vila Clementino, 04039-000, São Paulo, SP, Brasil. Correspondência para/Correspondence to: L. CUPPARI. E-mail: <lilian@dis.epm.br>.
} 
However, for chronic renal disease patients, this treatment is not completely effective, because although it promotes reduction of homocysteine levels, the normalization is not reached in the majority of the patients. This study reviews the hyperhomocysteinemia etiology on chronic renal disease, its main determinants, its relationship with vascular diseases, and the modes of treatment.

Indexing terms: homocysteine, hyperhomocysteinemia, kidney failure, chronic.

\section{N T R O D U Ç Ã O}

Vários estudos têm demonstrado que pacientes com insuficiência renal crônica (IRC) apresentam níveis elevados de homocisteína, incluindo os que se encontram na fase inicial da doença ${ }^{1}$. Por seus efeitos tóxicos ao endotélio, podendo causar trombose e/ou aterogênese, a hiperhomocisteinemia foi recentemente reconhecida como fator de risco independente para doença vascular, tanto em indivíduos não urêmicos quanto em pacientes com IRC. Em vista disso, a homocisteína tem sido amplamente estudada sob diversos aspectos, incluindo os mecanismos que podem estar envolvidos em sua ação aterogênica, as causas da alta prevalência de hiperhomocisteinemia em pacientes renais crônicos, e as terapias que podem auxiliar na sua prevenção e tratamento.

\section{METABOLISMO D A HO MOCISTEÍN A}

A homocisteína (Hcy) é um aminoácido sulfurado não formador de proteínas, sintetizada no metabolismo do aminoácido essencial metionina, proveniente tanto de fontes alimentares como do catabolismo de proteínas endógenas. A metionina condensa-se enzimaticamente com a porção adenosina da adenosina trifosfato (ATP), dando origem à S-adenosil-metionina (AdoMet). O produto da desmetilação da AdoMet é a S-adenosil-homocisteína (AdoHcy), único precursor da Hcy, por meio de uma reação irreversível catalisada pela AdoHcy hidrolase. A Hcy assim formada pode então seguir para duas vias: remetilação ou transulfuração (Figura 1$)^{2}$.

1) Remetilação: por esta via, a Hcy adquire um grupo metil para formar novamente a metionina, em uma reação catalisada pela metionina sintase, na qual a vitamina $\mathrm{B}_{12}$ age como cofator. O principal doador do grupo metil é o 5-metiltetrahidrofolato (5-MTHF), forma circulante ativa do folato. A Hcy pode, também, ser transformada em metionina pela ação da enzima betaína-homocisteína metiltransferase (BHMT), também dependente da vitamina $B_{12}$, que utiliza a betaína como doador de metil ${ }^{3}$. A reação com 5-MTHF ocorre em todos os tecidos, enquanto que a reação com betaína está confinada principalmente ao fígado e rins ${ }^{2}$.

2) Transulfuração: nesta via, a Hcy condensa-se com a serina para formar cistationina, por meio de uma reação irreversível, cuja enzima responsável é a cistationina beta sintetase (CBS), que tem como coenzima a forma ativa da vitamina $\mathrm{B}_{6}$, o piridoxal 5-fosfato (PLP). A cistationina é então hidrolisada à cisteína pela enzima cistationase, também dependente de PLP².

AdoMet é o principal regulador metabólico direcionador do fluxo de Hcy entre as vias de remetilação e transulfuração. Concentrações aumentadas de AdoMet, que refletem um excesso de metionina, provocam a inibição da enzima metilenotetrahidrofolato redutase (MTHFR), necessária para a formação do 5-MTHF, favorecendo assim, a via de transulfuração. Na presença de quantidades adequadas de metionina, aproximadamente $50 \%$ da Hcy é destinada à via de transulfuração4 .

Intracelularmente, a Hcy encontra-se principalmente em sua forma reduzida e seu transporte para o plasma é efetuado por meio de um carreador provavelmente específico. No plasma, apenas $1 \%$ encontra-se na forma reduzida, e $99 \%$ na forma oxidada. Destes, $20 \%$ combinam-se com compostos que contêm outro 
grupo tiol, como a própria Hcy (homodímeros) ou a cisteína (heterodímeros). A homocisteína reduzida, na forma de homo e heterodímeros, é denominada free Hcy (fHcy). Os $80 \%$ restantes da homocisteína oxidada encontram-se ligados a proteínas, principalmente à albumina, por meio de ligações dissulfídicas, sendo então denominadas bound Hcy (bHcy). Não estão esclarecidos quais dissulfídeos podem ser captados pela célula e quais tipos de transporte são requeridos. Após sua entrada na célula, a Hcy é liberada da ligação dissulfídica por redução, tornando-se disponível para ser metabolizada 5 .

Ainda existem controvérsias quanto aos níveis de Hcy que caracterizam a hiperhomocisteinemia. A classificação mais comumente encontrada é a seguinte: ${ }^{3}$

- Níveis normais de Hcy: entre 5 a 15 $15 \mathrm{~mol} / \mathrm{L}$;

- Hiperhomocisteinemia moderada: 16 a $30 \mu \mathrm{mol} / \mathrm{L}$
- Hiperhomocisteinemia intermediária: 31

a $100 \mu \mathrm{mol} / \mathrm{L}$;

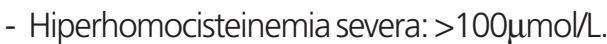

\section{HIPERHOMOCISTEINEMIA NA POPULAÇÃO EM GERAL}

A concentração plasmática da Hcy é determinada por fatores diversos, tanto genéticos como adquiridos. Audelin \& Genest citam os principais fatores que podem influenciar os níveis de Hcy na população ${ }^{6}$ :

1) Defeitos nos genes responsáveis pela codificação das enzimas envolvidas no metabolismo da Hcy: defeitos no gene codificador da cistationina beta sintetase (CBS) resultam na forma clássica de homocistenúria congênita, que se caracteriza por elevações na excreção urinária e nos níveis plasmáticos de Hcy, os quais se apresentam extremamente elevados nos

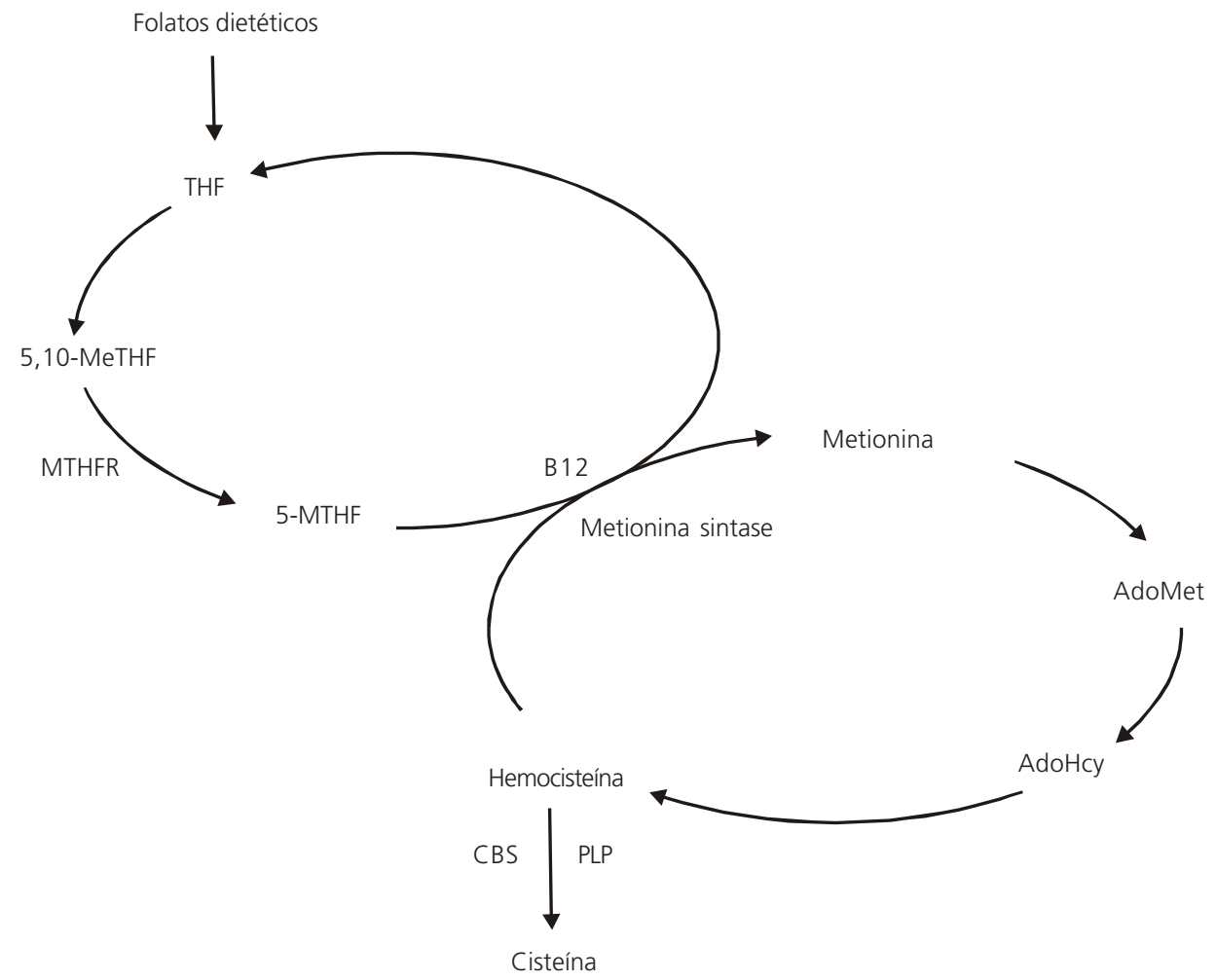

Figura 1. Metabolismo da homocisteína.

THF: tetrahidrofolato; 5,10-MeTHF: 5,10- metilenotetrahidrofolato; MTHFR: metilenotetrahidrofolato redutase; 5-MTHF: 5metiltetrahidrofolato; AdoMet: S-adenosil-metionina; AdoHcy: S-adenosil-homocisteína; CBS: cistationina beta sintetase; PLP: piridoxal 5fosfato. Adaptado de De Vriese et al. ${ }^{(34)}$. 
indivíduos homozigotos. Estes, geralmente, morrem antes de completarem 30 anos de idade, por algum evento tromboembólico agudo. Já nos indivíduos heterozigotos, a hiperhomocisteinemia é de grau leve a moderado ${ }^{6}$. Hiperhomocisteinemia menos severa pode também ser consequência de uma mutação do gene codificador da enzima metilenotetrahidrofolato redutase (MTHFR), responsável pela formação do 5-MTHF no ciclo do folato. Esta mutação pode ser homozigótica (TT) ou heterozigótica (CT) e ocorre devido à troca da citosina (C) por timina (T) no nucleotídeo 677 do gene codificador da MTHFR, resultando na substituição da alanina pela valina na enzima expressa. Isso confere à MTHFR uma menor atividade específica e uma maior sensibilidade à inativação por calor, por esta razão denominada MTHFR termolábil7. Quando comparados aos indivíduos que apresentam a variante genética normal (CC), a variação TT confere à enzima uma atividade sérica $70 \%$ menor, enquanto que para a variação $\mathrm{CT}$ a diminuição é de $35 \%{ }^{8}$.

A prevalência de indivíduos com mutação heterozigótica e homozigótica na população em geral parece depender da raça e varia entre as diferentes partes do mundo. Em estudo realizado com diferentes raças na população brasileira, Arruda et al. ${ }^{7}$ identificaram uma prevalência de variação homozigótica para a MTHFR em 10,00\% dos caucasianos, além de incidência em 1,45\% dos negros e em 1,20\% dos índios? ${ }^{7}$.

2) Idade: o envelhecimento pode levar ao aumento dos níveis de Hcy, tanto por redução do metabolismo enzimático como por alteração da função renal, ambas dependentes da idade ${ }^{6}$.

3) Sexo: os homens apresentam maiores níveis de Hcy do que as mulheres, provavelmente devido à maior massa muscular ou aos diferentes padrões hormonais. Porém, os níveis de Hcy das mulheres aproximam-se aos dos homens no período pós-menopausa ${ }^{6}$.

4) Deficiências nutricionais: deficiências das vitaminas envolvidas no metabolismo da Hcy, tais como folato, $\mathrm{B}_{6}$ e $\mathrm{B}_{12}$, também são relacionadas com os níveis desse aminoácido. Aproximadamente dois terços de todos os casos de hiperhomocisteinemia (baseados em amostras em jejum), estão associados com estado nutricional inadequado, por insuficiência de uma ou mais destas vitaminas. Isto sugere que, do ponto de vista de saúde pública, esta deficiência nutricional é a causa mais importante de níveis elevados de Hcy na população em geral².

5) Estilo de vida: além da dieta, outros fatores relacionados ao estilo de vida, tais como fumo, falta de atividade física, consumo de álcool e de café podem interferir nos níveis de Hcy. Porém, os dados disponíveis na literatura são ainda bastantes controversos. Em relação ao consumo de café e ao tabagismo, a maioria dos trabalhos realizados encontrou uma relação direta entre estas variáveis e a homocisteinemia ${ }^{9,10}$. De acordo com Mennen et al. ${ }^{11}$, a prática de atividade física parece influenciar de forma inversa os níveis de homocisteína. O papel do consumo de álcool difere entre os estudos, alguns mostrando uma relação direta ${ }^{11}$, e outros, inversa, com a homocisteinemia9.

6) Insuficiência renal crônica: está intimamente relacionada à hiperhomocisteinemia e será discutida a seguir.

\section{Homocisteína e doenças vasculares}

No final da década de 60, McCully fez a primeira observação clínica da relação entre níveis elevados de Hcy plasmática e doenças vasculares. O autor realizou autópsia em duas crianças com hiperhomocisteinemia e homocistenúria e encontrou vastas evidências de trombose arterial e aterosclerose. Com base nesta observação, o autor propôs que a hiperhomocisteinemia pode causar doença vascular aterosclerótica ${ }^{12}$.

A partir desse estudo, evidências epidemiológicas abundantes têm demonstrado que a hiperhomocisteinemia é um fator de risco independente para aterosclerose na vasculatura coronária, cerebral e periférica. Em trabalho 
realizado com indivíduos saudáveis e com pacientes com doença vascular previamente diagnosticada, os níveis de Hcy encontraram-se elevados em $42 \%$ dos pacientes com doença cerebrovascular, em $28 \%$ dos indivíduos com doença vascular periférica e em $30 \%$ dos que apresentavam doença vascular coronária. A prevalência de hiperhomocisteinemia nos indivíduos saudáveis foi nula. Neste mesmo estudo, os autores observaram que o risco relativo de doença coronária arterial em pacientes com hiperhomocisteinemia foi 24 vezes maior do que nos indivíduos saudáveis ${ }^{13}$.

\section{Mecanismos fisiopatológicos}

Apesar das inúmeras tentativas realizadas com o objetivo de elucidar o mecanismo pelo qual a Hcy contribui para complicações vasculares, este ainda não foi totalmente esclarecido. Mangoni \& Jackson ${ }^{3}$ citam os principais efeitos deletérios que parecem estar relacionados à ação aterogênica e trombogênica da Hcy. São eles: disfunção endotelial, espessamento da camada média das artérias, rigidez da parede arterial e atividade pró-coagulante.

\section{HO MOCISTEÍN A E I N S U F I C I Ê N C I A RENAL CRÔ N ICA}

Pacientes em diálise apresentam uma alta taxa de mortalidade, atribuída, principalmente, às doenças cardiovasculares; ademais, aproximadamente $40 \%$ dos pacientes que iniciam a terapia dialítica já apresentam evidências clínicas de doenças cardiovasculares. Porém, pouco é conhecido sobre a história natural da doença cardiovascular no curso da insuficiência renal. Segundo Perna et al. ${ }^{14}$, esta prevalência não deve ser atribuída apenas à presença de fatores de risco clássicos, como a hipertensão, o diabetes e as dislipidemias. Assim, há necessidade urgente do uso de terapias adjuntas, que tenham como alvo fatores de risco cardiovasculares não convencionais, como a hiperhomocisteinemia.

Apesar do conhecimento da relação entre hiperhomocisteinemia e falência renal ter expandido rapidamente em poucos anos, sua origem e fisiopatogenia não estão totalmente esclarecidas.

\section{Prevalência}

Vários estudos têm encontrado altos níveis de Hcy em pacientes com IRC em todas as fases da doença: não dialítica, dialítica e pós-transplante renal2,15,16.

Jungers et al. ${ }^{16}$ pesquisaram níveis de Hcy em 78 pacientes na fase não dialítica e obtiveram média de $21,2 \pm 6,4 \mu \mathrm{mol} / \mathrm{L}$ e uma prevalência de 95\% de hiperhomocisteinêmicos.

Os trabalhos realizados com a população em tratamento crônico de hemodiálise também encontraram uma prevalência de pacientes hiperhomocisteinêmicos, chegando a 100\% ${ }^{17,18}$. Nos pacientes em diálise peritoneal (DP), os níveis de Hcy são menores, quando comparados aos de pacientes em hemodiálise ${ }^{15}$.

Já em pacientes transplantados renais, são poucos os dados disponíveis na literatura sobre a prevalência e determinantes da hiperhomocisteinemia. Bostom \& Lathrop ${ }^{2}$ encontraram níveis de Hcy significantemente maiores em transplantados estáveis, quando comparados a indivíduos saudáveis.

\section{Homocisteína, Doenças Cardiovasculares e IRC}

Vários trabalhos na população de pacientes com IRC têm demonstrado a relação entre os níveis de Hcy e a presença e/ou risco de doenças cardiovasculares ${ }^{1,19}$.

Em pacientes com IRC não dialisados, Chauveau et al. ${ }^{1}$ comparou os níveis de Hcy entre os indivíduos com e sem história prévia de acidentes vasculares e observou que os primeiros 
apresentaram níveis de Hcy significantemente maiores $(30,9 \pm 19,1 \mu \mathrm{mol} / \mathrm{L}$ contra 19,6 \pm $9,7 \mu \mathrm{mol} / \mathrm{L})$. No trabalho realizado por Jungers et al. ${ }^{19}$, o nível de Hcy em pacientes na fase não dialítica foi um determinante independente para o desenvolvimento de acidentes cardiovasculares. O risco relativo para cada aumento de $1 \mu \mathrm{mol} / \mathrm{L}$ de Hcy foi de 1,17 (1,13-1,20; $p<0,001)^{1}$.

Ao contrário dos estudos descritos acima, o trabalho publicado recentemente por Suliman et al. ${ }^{20}$, que contou com a participação de 151 pacientes com IRC avançada (clearance de creatinina médio $\left.6,8 \pm 2,5 \mathrm{~mL} / \mathrm{min} / 1,73 \mathrm{~m}^{2}\right)$ que estavam prestes ( $33 \pm 7$ dias) ou logo após $(11 \pm 9$ dias) a entrada em tratamento dialítico, mostrou que os pacientes com sinais clínicos de doença cardiovascular (32\%) apresentavam níveis de Hcy significantemente menores $(29,0 \pm 14,9 \mu \mathrm{mol} / \mathrm{L})$ que os demais $(37,9 \pm 25,5 \mu \mathrm{mol} / \mathrm{L})$. Os autores explicam estes resultados pelo fato de os pacientes com história de doença cardiovascular serem mais desnutridos e apresentarem menores níveis de albumina sérica, dado que, por sua vez, foi o único preditor independente dos níveis de Hcy.

Em população em tratamento dialítico, um estudo prospectivo testou o poder preditivo da Hcy para eventos cardiovasculares em 175 pacientes em hemodiálise seguidos por $29 \pm 12$ meses; os autores concluíram que um aumento de $10 \mu \mathrm{mol} / \mathrm{L}$ nos níveis de Hcy plasmática correspondeu a uma elevação de 35\% no risco de evento aterotrombótico fatal ${ }^{21}$. Ainda em população em tratamento dialítico, o estudo realizado por Robinson et al..$^{22}$ concluiu que a hiperhomocisteinemia é um fator de risco independente para complicações ateroscleróticas. Porém, nem todos os trabalhos mostraram essa relação, sendo que alguns observaram níveis de Hcy significantemente menores em pacientes com doenças cardiovasculares. No trabalho de Suliman et al. ${ }^{18}$ os pacientes com níveis de Hcy $<24 \mu \mathrm{mol} / \mathrm{L}$, quando comparados àqueles com Hcy $>24 \mu \mathrm{mol} / \mathrm{L}$, além de apresentarem maior prevalência de doenças cardiovasculares, também tinham uma menor sobrevida. Os autores justificam este resultado pelo fato de a freqüência de desnutridos ser maior no grupo com doenças cardiovasculares.

De qualquer forma, a hiperhomocisteinemia parece ser um preditor importante de doenças vasculares, tanto na população em geral, como na população de pacientes renais crônicos.

\section{Determinantes dos níveis de homocisteína na IRC}

A hipótese de que o nível de filtração glomerular é o principal determinante dos níveis de Hcy tem sido demonstrada em estudos com pacientes renais crônicos na fase não dialítica, em que a taxa de filtração glomerular (TFG) está diminuída, e com diabéticos tipo I, cuja TFG está aumentada. Em ambos os casos, a TFG correlaciona-se inversamente com a homocisteinemia ${ }^{17,23}$.

Outros estudos em população não dialisada encontraram uma correlação significante e positiva entre níveis de Hcy e creatinina plasmática ${ }^{17}$, e negativa entre níveis de Hcy e depuração de creatinina ${ }^{24}$, reforçando essa hipótese.

Foram encontrados níveis elevados de Hcy em pacientes que apresentaram apenas uma leve redução na TFG; devido a esse fato, teme-se que o longo período de exposição aos efeitos deletérios da Hcy constitua um fator de risco importante para o aparecimento precoce da aterosclerose nos pacientes urêmicos ${ }^{1}$.

Como na população em geral, alguns fatores inatos, fisiológicos e comportamentais podem influenciar os níveis de Hcy nos pacientes renais crônicos. Alguns estudos ${ }^{20,25}$ encontraram como determinantes dos níveis de Hcy a albumina sérica, o sexo masculino ${ }^{21}$, e o equivalente protéico de aparecimento de nitrogênio (PNA) que estima a ingestão protéica ${ }^{18}$. Foi ainda encontrada relação inversa com o estado nutricional avaliado pela avaliação subjetiva global18,20. Não foram encontradas correlações com a idade e o hábito de fumar ${ }^{21}$. A presença ou não de diabetes mellitus 
parece não influenciar a homocisteinemia na população com IRC 23 .

A influência dos variantes genéticos da enzima MTHFR na hiperhomocisteinemia em renais crônicos também tem sido avaliada. Em um trabalho publicado recentemente, que caracterizou 168 pacientes em programa crônico de hemodiálise de acordo com o genótipo da MTHFR, observou-se que a variação homozigótica (TT) foi um forte determinante dos níveis de Hcy ${ }^{26}$. Esta mesma influência foi encontrada por outros autores ${ }^{25,27}$. Em relação às concentrações plasmáticas das vitaminas envolvidas no metabolismo da Hcy, alguns trabalhos mostraram que as concentrações de folato e de $B_{12}$ foram determinantes independentes dos níveis da Hcy ${ }^{25}$. Em outros, encontrou-se uma relação inversa entre níveis de Hcy e concentrações plasmáticas de folato, de vitamina $B_{12}{ }^{15,26,27}$ e de vitamina $B_{6}{ }^{15,24}$.

\section{Fisiopatologia da hiperhomocisteinemia na IRC}

Acreditava-se, antigamente, que a hiperhomocisteinemia na IRC era consequência da diminuição da excreção renal deste aminoácido. Porém, sabe-se, atualmente, que a excreção renal de Hcy é insignificante, pois 99\% sofrem reabsorção tubular. Em renais crônicos parece que esta via de excreção é um pouco maior, porém não chega a ser importante ${ }^{28}$. Assim, os principais fatores que parecem estar envolvidos com a fisiopatologia da hiperhomocisteinemia na IRC são:

- menor metabolismo renal da Hcy, como sendo o de maior importância;

- menor metabolismo extra-renal da Hcy, possivelmente devido ao meio urêmico;

- deficiência de vitaminas $B_{6}, B_{12}$ e folato, indispensáveis ao metabolismo da $\mathrm{Hcy}^{3}$.

A capacidade do rim em captar e metabolizar a Hcy é evidenciada pela habilidade do mesmo em filtrar moléculas pequenas, pela baixa concentração urinária deste aminoácido, pela presença de carreadores tubulares específicos e de todas as enzimas necessárias ao metabolismo da Hcy neste órgão 4 .

Em um importante estudo publicado sobre a cinética da Hcy em pacientes em programa crônico de hemodiálise, encontrou-se uma redução na depuração total de Hcy de aproximadamente $70 \%$, relacionada ao prejuízo na captação e no metabolismo desta pelos rins. Com isto, os autores sugerem que os rins são responsáveis por, pelo menos, $70 \%$ da depuração da Hcy plasmática em condições fisiológicas. Concluiu-se, portanto, que o acúmulo anormal de Hcy na IRC não é resultado de um aumento da sua liberação para o plasma, mas sim da sua prejudicada remoção do compartimento plasmático ${ }^{28}$.

Ainda não estão elucidadas em quais vias metabólicas específicas ocorre interferência da doença renal. Um trabalho que utilizou isótopos estáveis para o estudo do metabolismo da Hcy e da metionina em indivíduos em hemodiálise, mostrou que as vias de remetilação e transmetilação (formação Hcy a partir da metionina) estão significantemente diminuídas, quando comparadas às de indivíduos saudáveis. A via de transulfuração pareceu não estar afetada, mesmo com a observação de uma leve diminuição no grupo de pacientes. Portanto, os autores concluem que a diminuição na via de remetilação da Hcy é um fator importante para explicar a causa da hiperhomocisteinemia nesses pacientes, e que, provavelmente, a deficiência e/ou resistência ao folato e à vitamina $\mathrm{B}_{12}$ estejam envolvidas ${ }^{29}$.

Outro importante agravante que pode estar influenciando o acúmulo anormal da Hcy na IRC é a presença de substâncias urêmicas não identificadas que inibem o metabolismo extra-renal deste aminoácido. Acredita-se que pode haver presença de enzimas inibitórias e/ou menor quantidade de cofatores e substratos essenciais para o metabolismo da Hcy na uremia ${ }^{3}$.

\section{Tratamento}

Nos últimos anos, a comunidade científica vem realizando vários trabalhos com o intuito de 
esclarecer qual o melhor e mais efetivo tratamento para a redução dos níveis de homocisteína na população de pacientes renais crônicos. Para tanto, as vitaminas e outros compostos envolvidos no metabolismo deste aminoácido têm sido administrados nas diversas fases da IRC sob diferentes doses, formas e períodos de tratamento. Até os dias de hoje, o que se tem observado é que, na maioria dos estudos que utilizaram a suplementação com folato, $B_{6}$ e $B_{12}$, isoladas ou em conjunto, os níveis de Hcy reduzem em média $30 \%$, mas não se normalizam na maioria dos pacientes. Porém, é importante ressaltar que não há dados de estudos controlados e randomizados demonstrando se o tratamento bem sucedido da hiperhomocisteinemia é ou não capaz de reduzir o risco de doenças cardiovasculares nessa população.

Infelizmente, o tratamento da hiperhomocisteinemia na fase não dialítica tem sido pouco explorado, havendo poucos trabalhos na literatura sobre o assunto.

O primeiro estudo publicado foi em 1988, por Wilcken et al. ${ }^{30}$, em que 21 pacientes na fase não dialítica foram suplementados com $5 \mathrm{mg} / \mathrm{dia}$ de ácido fólico por um período que variou de 7 a 32 dias (duração média de $15 \pm 6$ dias). Em todos os pacientes, os níveis de Hcy livre diminuíram de $12,9 \pm 6,8 \mu \mathrm{mol} / \mathrm{L}$ para $6,8 \pm 2,8 \mu \mathrm{mol} / \mathrm{L}$ $(p<0,0001)$. Foi também observado que o grau de declínio da Hcy pós-suplementação estava positivamente relacionado com sua concentração inicial $(r=0,92)$. Este último achado foi também descrito por outros autores com pacientes em hemodiálise ${ }^{27}$.

Em um trabalho no qual 37 pacientes na fase não dialítica foram divididos em tercis, de acordo com o clearance de creatinina, foi fornecida suplementação de $70 \mathrm{mg} / \mathrm{dia}$ de piridoxina durante três meses e, em seguida, esta foi substituída por $10 \mathrm{mg} /$ dia de ácido fólico durante mais três meses. Os resultados mostraram que na pós-suplementação com piridoxina, não houve redução nos níveis de $\mathrm{Hcy}$, ao contrário dos resultados pós-suplementação de ácido fólico, cuja diminuição foi significante nos três grupos $(p<0,001)$. Os autores concluíram que doses farmacológicas de ácido fólico (mas não de piridoxina), são efetivas na diminuição de níveis elevados de Hcy, sugerindo, portanto, que o aumento da via de remetilação pode melhorar a hiperhomocisteinemia nesses pacientes ${ }^{31}$.

Em um protocolo de pesquisa 78 pacientes ambulatoriais com creatinina plasmática entre 1,5 e $7,2 \mathrm{mg} / \mathrm{dL}$ foram suplementados com $5 \mathrm{mg}$ de ácido fólico três vezes por semana, $250 \mathrm{mg}$ de piridoxina/semana e $1 \mathrm{mg}$ de vitamina $B_{12}$ duas vezes por semana, por um tempo que variou entre 12 e 74 meses (média 33,8 \pm 19,3 meses). Os níveis de Hcy total, folato e vitamina $B_{12}$ plasmáticos foram determinados no baseline (T0), após três meses (T3) e ao final do seguimento (Tf) de cada paciente. Os resultados obtidos demonstraram redução nos níveis de Hcy de 33\% no T3 e de $40 \%$ no Tf, mesmo com o aumento significante da creatinina plasmática e a diminuição do clearance de creatinina entre T0 e Tf. Além disso, do total de pacientes hiperhomocisteinêmicos (Hcy $>14,1 \mu \mathrm{mol} / \mathrm{L}$ ), no T0 (95\%), apenas $37 \%$ e $40 \%$ mantiveram-se com níveis elevados de Hcy no T3 e no Tf, respectivamente. Concluindo, a suplementação moderada das vitaminas parece ser fácil, de baixo custo, segura, e reduz substancialmente a hiperhomocisteinemia na fase pré-dialítica da IRC, com efeito sustentável por um longo período ${ }^{16}$.

No protocolo prospectivo, duplo-cego, de Thambyrajah et al. ${ }^{32}$ foram recrutados 100 pacientes em tratamento conservador com creatinina plasmática $>1,5 \mathrm{mg} / \mathrm{dL}$ e níveis de Hcy $>12 \mu \mathrm{mol} / \mathrm{L}$. Estes foram randomizados a receberem $5 \mathrm{mg} /$ dia de ácido fólico ou placebo, por 12 semanas. Após este período, a Hcy plasmática mostrou-se significantemente menor no grupo suplementado 15,1 (14,1 a 16,2 $\mu \mathrm{mol} / \mathrm{L})$ versus 20,1 (18,2 a 22,2 $\mu \mathrm{mol} / \mathrm{L})$. Estes valores representaram uma redução de $24,9 \%$ nos níveis de Hcy no grupo que recebeu ácido fólico. Além disso, 20,0\% dos pacientes do mesmo grupo conseguiram atingir homocisteinemia adequada. Concluindo, este estudo mostra que a dose 
administrada de ácido fólico diminuiu, mas falhou em normalizar os níveis de Hcy na maior parte destes pacientes.

Na população de pacientes renais crônicos submetidos a diálise, há vários trabalhos publicados sobre tentativas de tratamento da hiperhomocisteinemia.

Em um estudo multicêntrico, randomizado e duplo-cego, 144 pacientes em programa crônico de hemodiálise foram divididos em três grupos de acordo com a dose de ácido fólico que receberiam, 15, 30 ou $60 \mathrm{mg} /$ dia por quatro semanas. Os resultados mostraram um declínio nos níveis de Hcy já na primeira semana, que se manteve estável durante o período restante do tratamento. Os pacientes que receberam as doses de 15, 30 e 60mg/dia tiveram uma redução nos níveis de Hcy de 32,8\%, 29,9\% e 37,8\%, respectivamente, não havendo diferenças entre os grupos. Dos 120 pacientes com níveis elevados de Hcy antes do tratamento, apenas 30,8\% conseguiram normalizá-los ${ }^{33}$.

Outro estudo em pacientes em tratamento dialítico $(n=14)$, que apresentavam baixas concentrações de cobalamina sérica (<180pmol/ L) e níveis elevados de homocisteína, os mesmos foram suplementados com cianocobalamina intravenosa ( $1 \mathrm{mg} / \mathrm{semana}$ por 4 semanas). Após este período, os níveis de Hcy diminuíram em média 35\%. Os pacientes que não apresentavam o alelo T no genótipo da enzima MTHFR apresentaram níveis de Hcy significantemente menores que os demais. $\mathrm{O}$ efeito adverso deste tratamento foi a queda observada nos níveis de folato plasmático de $47 \%$. Os autores supõem que este fato pode ter ocorrido devido à maior captação celular do 5-MTHF diminuindo, portanto, a concentração sérica desta vitamina. Concluindo, como a suplementação com $B_{12}$ influenciou negativamente os níveis de folato, é necessária a administração conjunta destas vitaminas ${ }^{27}$.

Como na população de pacientes não dialisados, nota-se uma resistência aos diversos tipos de tratamento com suplementação vitamínica testados, já que apenas uma minoria dos pacientes atinge níveis adequados de homocisteína. Algumas justificativas para que este fato ocorra são os distúrbios do metabolismo do folato na presença da uremia ${ }^{29}$ e a saturação dos níveis desta vitamina pós-suplementação ${ }^{34}$. Além disso, se os rins exercem o principal papel no metabolismo da Hcy, então o parênquima renal não funcionante não poderia responder significantemente à suplementação vitamínica, não importando quão intensa ela seja ${ }^{34}$.

Ainda não existe um consenso quanto à dose e ao modo de administração ideais da suplementação vitamínica para o tratamento da hiperhomocisteinemia nos pacientes com IRC. Em revisão publicada recentemente, avaliando inúmeros trabalhos sobre o assunto, os autores concluíram que a suplementação vitamínica que tem obtido os melhores efeitos na população de pacientes renais crônicos é a de um suplemento oral e diário que contenha de 1 a 5mg/dia de ácido fólico, 6 a $12 \mu \mathrm{g}$ de vitamina $B_{12}$ e 10 a 50mg de vitamina $\mathrm{B}_{6}{ }^{34}$.

\section{REFERÊ NCIAS}

1. Chauveau P, Chadefaux B, Coude M, Aupetit J, Hannedouche T, Kamoun $P$, et al. Hyperhomocysteinemia, a risk factor for atherosclerosis in chronic uremic patients. Kidney Int. 1993; 41:S72-7.

2. Bostom AG, Lathrop L. Hyperhomocysteinemia in end-stage renal disease: Prevalence, etiology, and potential relationship to arteriosclerotic outcomes. Kidney Int. 1997; 52(1):10-20.

3. Dennis $\vee$, Robinson K. Homocysteinemia and vascular disease in end-stage renal disease. Kidney Int. 1996; 50:S11-7.

4. Blom HJ, De Vriese S. Why are homocysteine levels increased in kidney failure? A metabolic approach. J Lab Clin Med. 2002; 139(5):262-8.

5. Mangoni AA, Jackson SHD. Homocysteine and cardiovascular disease: Current evidence and future prospects. Am J Med. 2002; 112(7):556-65.

6. Audelin MC, Genest Jr J. Homocysteine and cardiovascular disease in diabetes mellitus. Atherosclerosis. 2001; 159(2):497-511. 
7. Arruda VR, Siqueira LH, Gonçalves MS, von Zuben PM, Soares MC, Menezes R, et al. Prevalence of the mutation C677à $T$ in the methylene tetrahydrofolate reductase gene among distinct ethnic groups in Brazil. Am J Med Genet. 1998; 78(4):332-5.

8. Frosst P, Blom HJ, Milos R, Goyette P, Sheppard CA, Mathews RG, et al. A candidate genetic risk factor for vascular disease: a common mutation in methylenetetrahydrofolate reductase. Nat Genet. 1995; 10(1):111-3.

9. De Bree A, Verschuren WMM, Blom HJ, Kromhout D. Lifestyle factors and plasma homocysteine concentrations in a general population sample. Am J Epidemiol. 2001; 154(2):150-4.

10. Jackes PF, Bostom AG, Wilson PW, Rich S, Rosenberg $\mathrm{IH}$, Selhub J. Determinants of plasma total homocysteine concentration in the Framingham Offspring cohort. Am J Clin Nutr. 2001; 73(3): 613-21.

11. Mennen LI, de Couray GP, Guilland JC, Ducros V, Bertrais S, Nicolas JP, et al. Homocysteine, cardiovascular disease risk factors, and habitual diet in the French Supplementation with Antioxidant Vitamins and Minerals Study. Am J Clin Nutr. 2002; 76(6):1279-89.

12. Welch GH, Loscalzo J. Homocysteine and atherothrombosis. N Eng J Med. 1998; 338(15): 1042-50.

13. Clarke R, Daly L, Robinson K, Naughten E, Cahalane S, Fowler B, et al. Hyperhomocysteinemia: An independent risk factor for vascular disease. $\mathrm{N}$ Engl J Med. 1991; 324(17):1149-55.

14. Perna $A F$, Ingrosso $D$, Satta $E$, Romano $M$, Cimmino A, Galletti P, et al. Metabolic consequences of hyperhomocysteinemia in uremia. Am J Kidney Dis. 2001; 38(4 Suppl 1):S85-90.

15. Moustapha A, Grupta A, Robinson H, Arheart K, Jacobson DW, Schreiber MJ, et al. Prevalence and determinants of hyperhomocysteinemia in hemodialysis and peritoneal dialysis. Kidney Int. 1999; 55(4):1470-5.

16. Jungers $P$, Joly $D$, Chauveau $P$, Nguyen $A T$, Aupetit J, Chadefaux, B. Sustained reduction of hyperhomocysteinemia with folic acid supplementation in predialysis patients. Nephrol Dial Transplant. 1999; 14(12):2903-6.

17. Hultberg B, Andersson A, Sterner G. Plasma homocysteine in renal failure. Clin Nephrol. 1993; 40(4):230-4.

18. Suliman ME, Qureshi AR, Barany P, Stenvinkel P, Filho JC, Anderstam B, et al. Hyperhomocysteinemia, nutritional status, and cardiovascular disease in hemodialysis patients. Kidney Int. 2000; 57(4): 1727-35.
19. Jungers P, Massy ZA, Khoa TN, Fumeron C, Labrunie $M$, Lacour $B$, et al. Incidence and risk factors of atherosclerotic cardiovascular accidents in predialysis chronic renal failure patients: a prospective study. Nephrol Dial Tranplant. 1997; 12(12):2597-602.

20. Suliman $M E$, Stenvinkel $P$, Heimbürger $O$, Bàràny $P$, Lindholm B, Bergström J. Plasma sulfur amino acids in relation to cardiovascular disease, nutritional status, and diabetes mellitus in patients with chronic renal failure at start of dialysis therapy. Am J Kidney Dis. 2002; 40(3):480-8.

21. Malamaci F, Zoccali C, Tripepi G, Ferme I, Benedetto FA, Cataliotti A, et al. Hyperhomocysteinemia predicts cardiovascular outcomes in hemodialysis patients. Kidney Int. 2002; 61(2):609-14.

22. Robinson K, Grupta A, Dennis V, Arheart K, Chaudhary D, Green R, et al. Hyperhomocysteinemia confers an independent increase risk of atherosclerosis in end-stage renal disease and is closely linked to plasma folate and pyridoxine concentrations. Circulation. 1996; 94(11): 2743-78.

23. Wollesen F, Brattström L, Refsum $H$, Ueland PM, Berglund L, Berne C. Plasma total homocysteine and cysteine in relation to glomerular filtration rate in diabetes mellitus. Kidney Int. 1999; 55(3):1028-35.

24. Arnadottir M, Hultberg B, Nilsson-Ehle P, Thysell $H$. The effect of reduced glomerular filtration rate on plasma total homocysteine concentration. Scand J Lab Invest. 1996; 56(1):41-6.

25. Wrone EM, Zehnder JL, Hornberger JM, McCann LM, Coplon NS, Fortmann SP. An MTHFR variant, homocysteine, and cardiovascular comorbidity in renal disease. Kidney Int. 2001; 60(3):1106-13.

26. Morimoto K, Haneda T, Okamoto K, Ishida H, Kikuchi K. Methylenetetrahydrofolate reductase gene polymorphism, hyperhomocysteinemia, and cardiovascular diseases in chronic hemodialysis patients. Nephron. 2002; 90(1):43-50.

27. Dierkes J, Domröse U, Bosselmann HP, Neumann $\mathrm{KH}$, Luley C. Response of hyperhomocysteinemia to folic acid supplementation in patients with end-stage renal disease. Clin Nephrol. 1999; 51(2):108-15.

28. Guttormsen $A B$, Ueland PM, Svarstad E, Refsum $\mathrm{H}$. Kinetcs basis of hyperhomocysteinemia in patients with chronic renal failure. Kidney Int. 1997; 52(2):495-502.

29. Van Guldener C, Kulik W, Berger R, Dijkstra DA, Jackobs C, Reyngoud DJ, et al. Homocysteine and methionine metabolism in ESRD: A stable isotope study. Kidney Int. 1999; 56(3):1064-71. 
30. Wilcken DEL, Budman NPB, Tyrrell PA, Robertson MR. Folic acid lowers elevated plasma homocysteine in chronic real insufficiency: Possible implications for prevention of vascular disease. Metabolism. 1998; 37(4):697-701.

31. Chauveau P, Chadefaux B, Coude M, Aupetit J, Kamoun $P$, Jungers $P$. Long-term folic acid (but not pyridoxine) supplementation lowers elevated plasma homocysteine level in chronic renal failure. Miner. Eletrolyte Metab. 1996; 22(1-3):106-9.

32. Thambyrajah J, Landray MJ, McGlynn FJ, Jones HJ, Wheeler DC, Townend JN. Does folic acid decrease plasma homocysteine and improve endothelial function in patients with predialysis renal failure? Circulation. 2000; 102(8):871-5.
33. Sunder-Plassmann $G$, Foolinger $M$, Buchmayer $H$, Papagiannopaulos M, Woja KJ, et al. Effect of high dose folic acid therapy on hyperhomocysteinemia in hemodialysis patients: Results of the Vienna Multicenter Study. J Am Soc Nephrol. 2000; 11(6): 1106.

34. De Vriese AS, Verbeke F, Brieke FS, Lameire NH. Is folate a promising agent in the prevention and treatment of cardiovascular disease in patients with renal failure? Kidney Int. 2002; 61(4): 1199-209.

Recebido para publicação em 13 de junho e aceito em 16 de novembro de 2003. 